\section{EMBRYRIDDLE \\ Aeronautical University}

SCHOLARLY COMMONS

\section{International Journal of Aviation,} Aeronautics, and Aerospace

\title{
A Review of DJI's Mavic Pro Precision Landing Accuracy
}

Cody Yoakum

Embry-Riddle Aeronautical University, g.cody.yoakum@gmail.com

Joseph Cerreta

Embry-Riddle Aeronautical University, cerretaj@erau.edu

Follow this and additional works at: https://commons.erau.edu/ijaaa

Part of the Aeronautical Vehicles Commons, and the Aviation Safety and Security Commons

\section{Scholarly Commons Citation}

Yoakum, C., \& Cerreta, J. (2020). A Review of DJI's Mavic Pro Precision Landing Accuracy. International Journal of Aviation, Aeronautics, and Aerospace, 7(4). https://doi.org/10.15394/ijaaa.2020.1524

This Article is brought to you for free and open access by the Journals at Scholarly Commons. It has been accepted for inclusion in International Journal of Aviation, Aeronautics, and Aerospace by an authorized administrator of Scholarly Commons. For more information, please contact commons@erau.edu. 


\section{A Review of DJI's Mavic Pro Precision Landing Accuracy}

For a relatively inexpensive, over-the-counter Unmanned Aircraft System (UAS), the DJI Mavic Pro comes standard equipped with unique sensors and proprietary algorithms to assist in the overall automation and ease of use (DJI, 2017). This design opened the potential use of the Mavic Pro from initial hobby introduction to commercial UAS operations by capturing high-resolution images and video (Zhang \& Kovacs, 2012; Zhong et al., 2018). One distinctive function of the Mavic Pro is its ability to conduct a "Precision Landing," assisting the device to autonomously land when specific terrain features match a recorded takeoff image from its downward positioning cameras (DJI, 2017). Precision landings can be beneficial for safety, efficiency, or unique mission specifications (Borowczyk et al., 2017; Kaljahi et al., 2019; Rohan et al., 2018). For example, Rohan et al. (2018) found that autonomous operations are less effective when drones cannot land precisely within $100 \mathrm{~mm}$ of a target when conducting wireless battery charging.

There was limited information published from DJI that defined the accuracy of the Mavic Pro's precision landing. There was also limited publicly available data for determining the accuracy of a Mavic Pro's landing accuracy while using either precision landing or a non-precision automated landing. The user manual describes the process of a precision landing as automatically scanning and attempting to match terrain features underneath the UAS when Return to Home has been activated (DJI, 2017). If the terrain matches, then the landing will commence automatically, otherwise depending on the software or environmental variables, the device will wait for pilot intervention (DJI, 2017). This action was predicated on capturing a "precision takeoff image" shortly after the aircraft lifts off and reaches 19.6 feet (Figure 1). The "precision takeoff enables" is what the UAS uses to compare the landing zone terrain features (DJI, 2017). 


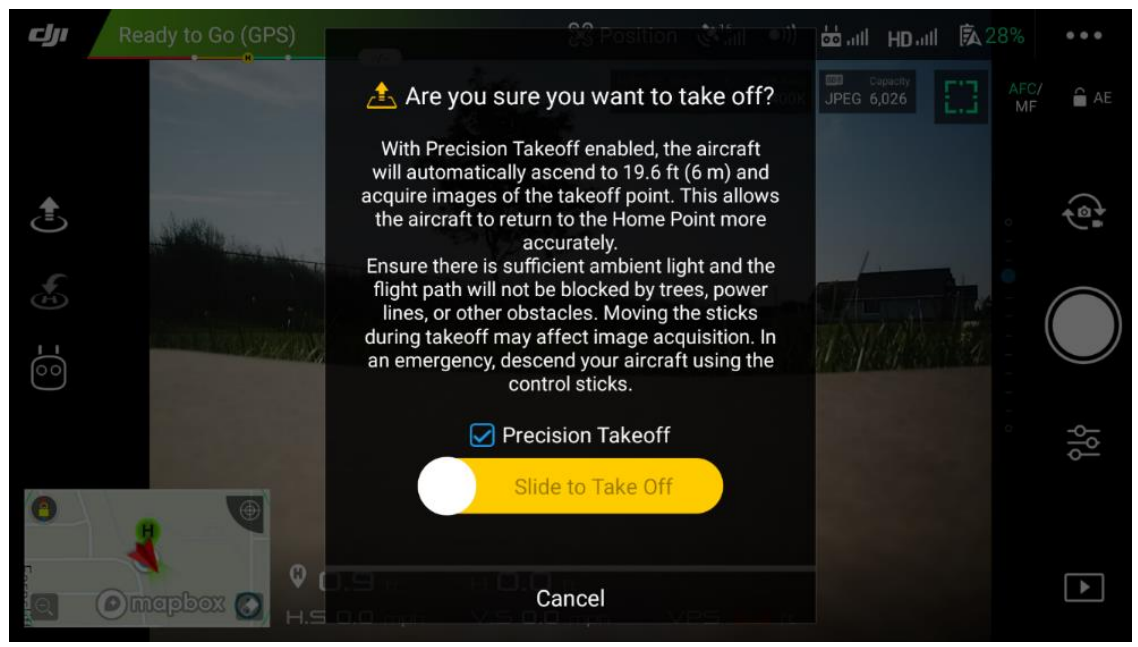

Figure 1. Screenshot of the DJI GO4 Precision Takeoff instructions before takeoff.

By calculating and defining the performance landing capability, more precise landing demands, such as landing for wireless charging or launching/landing in confined spaces, can be obtained (Kaljahi et al., 2019; Rohan et al., 2018). In contrast, pilots may choose to deactivate the precision landing function if critical sensors required for a precision landing are damaged (DJI, 2017). By deactivating the precision landing function, an operator still receives aid with autonomous landings; however, the landings may not be as precise and could require the pilot's confirmation to execute the final touchdown (DJI, 2017). Understanding the strengths and limitations of what a device can assist in adequately sourcing the device for the best mission while increasing safety and efficiency (Kaljahi et al., 2019; Rohan et al., 2018).

\section{Purpose}

The DJI Mavic Pro has no published information on the accuracy/dimensions of a landing point. Autonomous landings maneuver the aircraft to comply with either a GPS waypoint/precision landing terrain match or based on the last recorded Home Point from GPS signal before takeoff (DJI, 2017). It is unknown if precision landings are more precise or require a smaller landing space than non-precision landing. This research aims to investigate two research questions; "Do precision landing systems with a DJI Mavic Pro improve autonomous landing accuracy?" It is also unknown if the DJI Mavic Pro precision landings can meet the $100 \mathrm{~mm}$ tolerance required for wireless battery charging; "Can a DJI Mavic Pro autonomously land with enough accuracy to enable wireless charging?" In answering these questions, the DJI Mavic Pro may be capable of 
specific applications that require landing within a confined space (e.g., wireless charging, emergency landings, small launch pads; Borowczyk et al., 2017; Kaljahi et al., 2019; Rohan et al., 2018).

\section{Review of Relevant Literature}

Although not specific to the DJI Mavic Pro, the potential use of examining, defining, and improving precision landings to near $100 \%$ accuracy can have significant consequences. Autonomous landings have proven to be a challenging problem for the robotic community and continue to require additional study (Nguyen et al., 2018). Research needs to focus on onboard, autonomous functions that improve a UAS's functionality to open the possibility of a broader range of applications (Kyristsis et al., 2016). Selected relevant literature focuses on optical estimations and identifications, tracking and landing on moving platforms, and practical applications involving wireless battery charging and emergency landing locations.

\section{Optical Altitude Estimation and Marker Identification}

It was vital for UAS to land in predefined areas accurately and safely, as this phase of flight was most prone to accidents (Yang \& Sun, 2018). Traditionally, the global positioning system (GPS) and inertial navigation systems (INS) have been used to assist with UAS landings. However, GPS is not always available in all areas, or when it is, ordinary GPS systems can have errors as large as one meter (Yang \& Sun, 2018). Inertial measurement units (IMU) typically consist of a threeaxis gyroscope, accelerometer, and magnetometer and is an essential component of the INS (Yang \& Sun, 2018). Unfortunately, a gyroscope is inaccurate because of $\mathrm{drift} /$ noise (Yang \& Sun, 2018). Simultaneously, the accelerometer is too sensitive to dynamic motion, and a magnetometer can receive interference from electrical noise from daily appliances and devices (Yang \& Sun, 2018).

As a result of the helpful but flawed GPS/INS sensors, visual/optical navigation for UAS landings has received increased attention (Yang \& Sun, 2018). Visual navigation is relatively inexpensive, uninterrupted, and passive (Yang \& Sun, 2018). These optical systems can provide navigation, obstacle avoidance, and altitude information (Yang \& Sun, 2018). Three main drawbacks to optical systems deal with insufficient light, blurred images (smudged, blocked, or dirty lens), and difficulty when the background image is indistinguishable (water, grass, or sand); (Yang \& Sun, 2018; Kaljahi et al., 2019). Specific to landings, optical systems provide altitude estimation using iterative methods like particle filtering, Kalman filtering, and a gradient descent algorithm (Yang \& Sun, 2018). Traditionally the Kalman filter is used but provides a drawback that directly estimating attitude can cause a divergence for non-linearized UAS (Yang \& Sun, 2018).

The research consisted of an optical camera and a Kalman filter to complement the IMU sensor (Yang \& Sun, 2018). The camera pointed down so 
that the yaw angle could be provided from the vision system, complimenting the yaw data provided by the magnetometer (Yang \& Sun, 2018). Data was filtered and compared between the vision system, magnetometer, accelerometer, and gyroscopic compensations (Yang \& Sun, 2018). A stm32 and Raspberry Pi were attached to a UAS for image processing and autonomous landing experiments (Yang \& Sun, 2018). A direct result of the simulations and experiments showed that the proposed algorithms and sensors guaranteed rapid dynamic response, with the proposed algorithm showing better performance than traditional methods (Yang $\&$ Sun, 2018). This research directly contributes to the attitude estimation of UAS in autonomous landings.

Further research into altitude estimation demonstrated that automatic landings of UAS were non-trivial tasks (Miller et al., 2019). There was an emphasis that the magnitude of measurement errors related to current altimeters was unacceptably high for controlling landing maneuvers (Miller et al., 2019). It was desirable to have another means of altitude estimation, with the optical flow (OF) being a suitable candidate (Miller et al., 2019). Various uses of OF in UAS applications included landing at unknown hazardous environments, landing with the aid of landing pads, vison-based mapping for landing, and landing maneuvers in a UAS (Miller et al., 2019). One challenge with OF lies in the susceptibility of the camera resolution to the shift rate of the recorded image (Miller et al., 2019). For example, during the descent phase of flight, it was noticed that the designated parameters could change at least one hundred times with the change in altitude (Miller et al., 2019). The rate of image motion changes led to the degradation of the resolution, making the detection of landmarks difficult (Miller et al., 2019).

Additional research into vision systems focused on developing specific markers to assist with location recognition and autonomous landing tasks (Nguyen et al., 2017). Amazon's UAS service, Prime Air, was known to use an Amazonbranded landing pad as a beacon for their unmanned aircraft to detect, land, and deliver the payload (Nguyen et al., 2017). This additional research proposed a novel algorithm for marker identification and tracking for autonomous landings (Nguyen et al., 2017).

Nguyen et al. (2017) researched locating and tracking a target (landing pad) for autonomous drone landings, which focused on the landing control of the unmanned aircraft. The marker had a width and height of 1 meter and comprised three inner circles, divided into eight areas with an even distribution of white and black sections (Nguyen et al., 2017). The segment from $0^{\circ}$ to $45^{\circ}$ was inverse color compared to the rest of the marker, to assist with direction identification (Nguyen et al., 2017).

The marker image was broken down into simple geometric lines/circles based on color detection of white and black (Nguyen et al., 2017). Connected points created diameters within the circle, and intersecting diameters identified the center 
of the circle (Figure 2; Nguyen et al., 2017). This algorithm works for both day and night operations (Nguyen et al., 2017). Adaptive thresholding was applied, followed by a Hit and Miss morphology, which produced a pixelated image of the marker that assisted with identification in night conditions (Nguyen et al., 2017). The proposed algorithm used these low-quality images to identify the center point on the marker (Nguyen et al., 2017). In their experiments, a DJI Phantom 4 quadcopter was used, with captured colored images converted into gray ones to provide for faster computational processing (Nguyen et al., 2017).

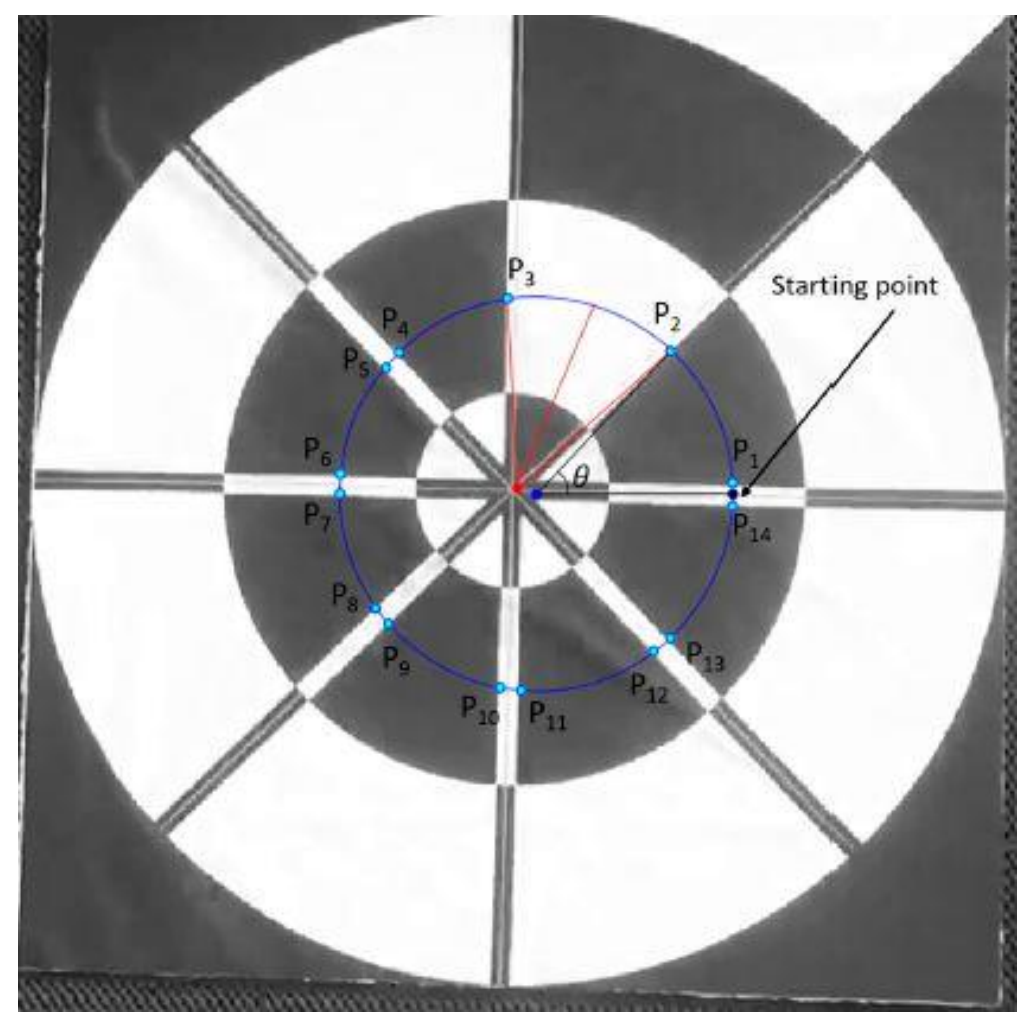

Figure 2. The customized marker with an overlay of the prediction center and direction (Nguyen et al., 2017).

There were no open databases of images of drones performing landings operations, so Nguyen et al. (2017) captured eight videos in a variety of conditions to provide the requisite image base for their algorithm testing. The morning, afternoon, and evening experiments flew at 6 and 10 meters, whereas night flights were undertaken only at 6 meters because the camera could not detect the marker (Nguyen et al., 2017). At 10 meters, scale variation and having a cluttered 
background created challenging factors for their system (Nguyen et al., 2017). Overall, the proposed algorithm proved to successfully identify the target and identified the center more accurately than traditional means (Nguyen et al., 2017). Tracking and Landing on Moving Platforms

The relatively short battery life of a quadcopter design limited the range the UAS could operate (Borowczyk et al., 2017). A potential solution was to launch and recover the unmanned aircraft from a mobile ground vehicle (Borowczyk et al., 2017). Two issues arose from mobile platforms, the size of the landing pad and velocity estimation (Borowczyk et al., 2017). The landing pad dimensions were confined and finite (e.g., the width of a truck bed/roof of a car). This concept extended to maritime operations, where ship dimensions were also limited (Borowczyk et al., 2017). Some systems rely upon optical flow for velocity estimation (Borowczyk et al., 2017). At a point, the UAS loses reference to the surrounding environment (e.g., the ground is out of the camera field of view) and measures its velocity relative to the platform (Borowczyk et al., 2017).

Low-quality sensors provided enough data to enable the ground vehicle and the UAS to communicate spatial localization information (Borowczyk et al., 2017). A camera mounted on the UAS provided feedback based on viewing the AprilTag located on the landing platform. Simultaneously, a mobile device sends GPS, headings, and inertial measurement unit data information relative to the landing pad (Borowczyk et al., 2017). With the use of a Kalman filter to estimate the position of the landing pad concerning the UAS and basic over-the-counter sensors successfully allowed the device to land on the moving vehicle at speeds up to 50 $\mathrm{km} / \mathrm{h}$ (Borowczyk et al., 2017). There was no reference on the accuracy of the landing point, where the device touched down, how frequently it landed on the point, or to the overall size of the landing pad. The AprilTag was $30 \times 30 \mathrm{~cm}$ (Borowczyk et al., 2017).

The overall size and velocity of a moving platform were determinative factors in the success of previous approaches (Kyristsis et al., 2016). The research focused on improving visual marker detection, accurate target geolocation, and the implementation of an "aggressive," "follow," and "hybrid" approach mode (Kyristsis et al., 2016). There was an emphasis on applying this research towards low-cost, small, personalized UAS uses (Kyristsis et al., 2016).

Further research added two additional axes (pose and heave) to the concept of a moving platform, with research into landing UAS on a vessel deck (Wang \& Bai, 2017). The most considerable challenge to ship deck landing is disturbances from ocean waves', compounded by expected constant winds (Wang \& Bai, 2017). Challenges related to previous moving platform landings still apply to this situation (error-ridden sensors, complex algorithms, limitations on speed; Wang \& Bai, 2017). 
Additional work was noted that landing on a moving platform was possible by using deep deterministic policy gradients (DDPG) algorithms for both simulated and actual flights (Rodriguez-Ramos et al., 2018). The DDPG process hinged on deep reinforcement learning theory that allowed a device to balance exploration and exploitation of the state space; the device was "rewarded" for successful actions (Rodriguez-Ramos et al., 2018). In testing, there were a few instances where reinforcement learning required the simulation clock to be stopped due to computationally demanding training steps (Rodriguez-Ramos et al., 2018).

By rewarding the system for successful actions, the UAS learned to minimize position differences concerning the moving platform, while also monitoring the velocity difference between the drone and moving platform (Rodriguez-Ramos et al., 2018). The UAS first learned to coarsely minimize the differences, with additional training resulting in optimized behavior with smoother velocity corrections (Rodriguez-Ramos et al., 2018). One assumption in practice was that the position and velocity of both the drone and moving platforms were always known (Rodriguez-Ramos et al., 2018).

\section{Practical Applications}

One common challenge with using electrically powered UAS was endurance (Rohan et al., 2018). Quadcopter UAS designs provided the opportunity for vertical takeoff and landing, hovering, and maneuvering in tight corridors; however, because of the power required to maintain lift, these unmanned aircraft typically had low endurance and needed frequently changed batteries (Rohan et al., 2018).

Previous research showed the potential of using wireless power transmission to charge UAS batteries with little to no required human interaction (Rohan et al., 2018). The system used electromagnetic coils on both the quadcopter and base station, which required both sets of coils to be in alignment for efficient power transfer (Rohan et al., 2018). Because of the unfortunate landing effect of quadcopters in general, the misalignment of the coils caused power loss and affected the overall efficiency of the charging system (Rohan et al., 2018). As a result of imperfect landings, the researchers designed a wireless battery charging base station, where an internal platform would position the base coils underneath a landed drone (Rohan et al., 2018). Instead of focusing on getting the unmanned aircraft to land precisely on the coils, the base station would prevent any misalignment during power transmission (Rohan et al., 2018). The overall design allowed for the efficiency level of up to $85 \%$ from a system landing accuracy of 98.8\% (Rohan et al., 2018).

Further research into the practice of precision landings could prove valuable when a UAS was operating in confined spaces or during critical emergency scenarios. Kaljahi et al. (2019) demonstrated the potential for onboard systems to evaluate the surrounding environment and determine relatively safe landing areas 
for potential use. The overall system architecture relied on a triggered fault detection (e.g., poor weather conditions, obstacles, internal failure) that activated the diagnostics, possible courses of action/zone detection and determinization, and then conducting a safe landing (Kaljahi et al., 2019). The system used the Gabor Transformation on captured images and compared histograms from different photos. The transformation grouped pixels into regions that could be used as a reference point to calculate which part was safe for landing (Kaljahi et al., 2019). Occasionally, the proposed area was a false candidate region, mainly when a distinct textural background was unavailable (e.g., water was most of the background environment or environmental conditions that blurred the camera; Kaljahi et al., 2019). Using small and medium UAS, the proposed process outperformed previous practices in detecting safe landing areas (Kaljahi et al., 2019).

\section{Method}

Two field data collections were used in this research. Field Collection 1 (difference in precision landing on/off) supported to Hypothesis 1, while Field Collection 2 (landing deviation within 100mm) supported with Hypothesis 2. The research questions (RQ) and hypotheses were:

RQ1: Do precision landing systems with a DJI Mavic Pro improve autonomous landing accuracy?

$\mathrm{H} 1_{0}$ : There is no statistical difference in landing accuracy when landing with "precision landing" on vs. off.

$\mathrm{H} 1_{1}$ : There is a statistical difference in landing accuracy when landing with "precision landing" on vs. off.

RQ2: Can a DJI Mavic Pro autonomously land with enough accuracy to enable wireless charging?

$\mathrm{H} 2_{0}$ : A DJI Mavic Pro can consistently land within $100 \mathrm{~mm}$ of its designated landing location for wireless charging.

$\mathrm{H} 2_{1}$ : A DJI Mavic Pro cannot consistently land within $100 \mathrm{~mm}$ of its designated landing location for wireless charging.

These two investigations were similar in that they both could assist in calculating landing accuracy. Each provided separate specifications that may support future research. Field Dataset 1 highlighted the potential benefit of precision landings, while Field Dataset 2 provided a margin of error when comparing landing positioning from the original takeoff location. Both datasets were collected at an open field in Caldwell, ID on August 21-22, 2020, as depicted in Figure 3. 


\section{Research Question \#1}

Comparing the "precision landing" function determined the differences in accuracy with and without the precision landing capability enabled. A two-tail, twosample t-test statistically compared the mean distances between landings when the precision landing was activated or not activated. A total sample size of 128 data points (64 takeoffs and landings with precision landing on/off each) were recorded.

\section{Research Question \#2}

Data collection focused on determining the overall landing accuracy (precision landing on) of the DJI Mavic Pro. An average landing accuracy could help determine future applications, as the "minimum required landing area dimensions" (Borowczyk et al., 2017; Kaljahi et al., 2019; Rohan et al., 2018). A one-tailed, one-sample t-test was used to determine if the DJI Mavic Pro could consistently land within a $100 \mathrm{~mm}$ radius from the initial starting takeoff point; a $100 \mathrm{~mm}$ radius represented the distance when efficiency decreased in wireless charging (Rohan et al., 2018). A total sample size of 64 data points were recorded. 


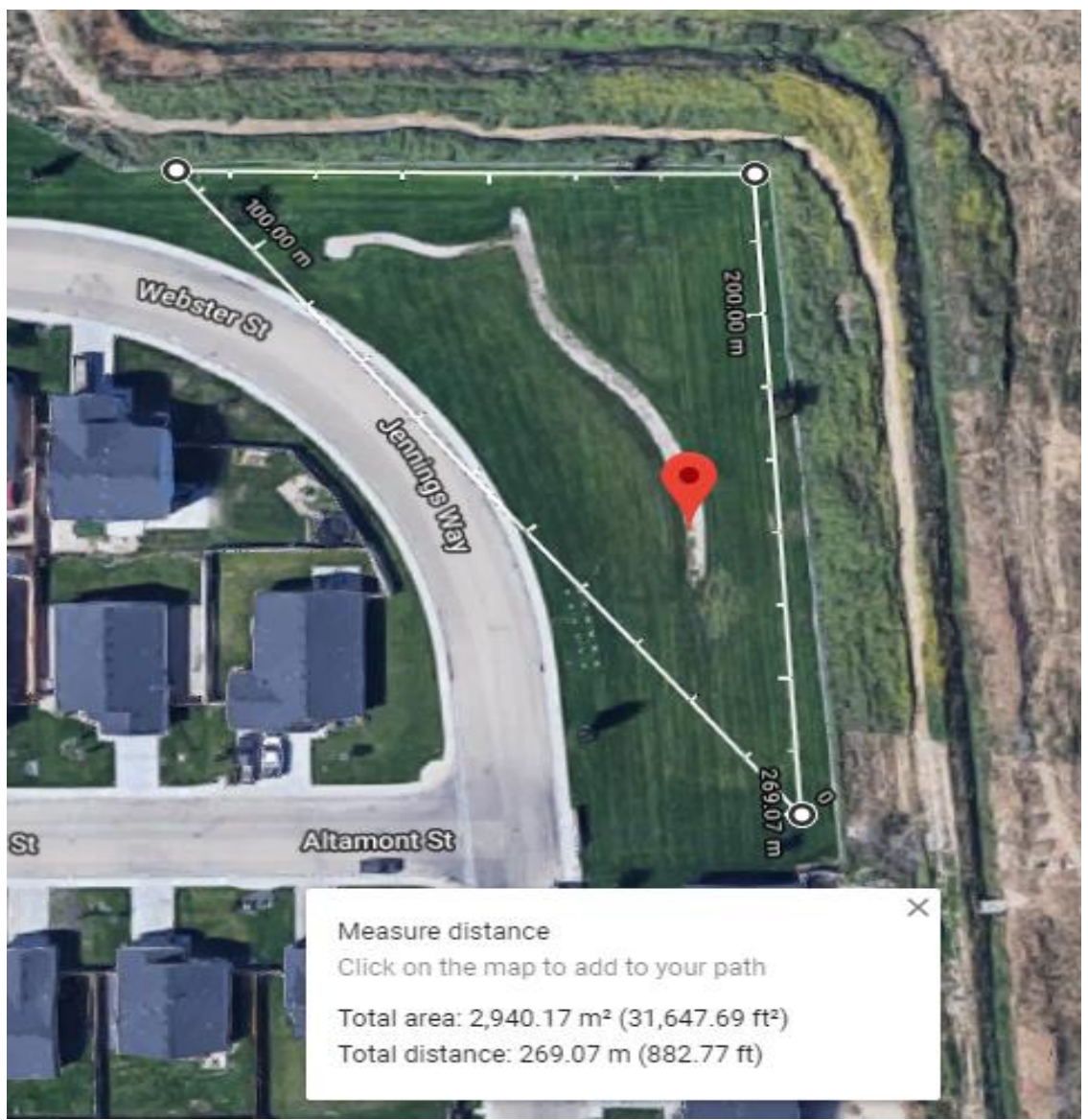

Figure 3. The flight operations area where data collection occurred, located in Caldwell, ID. (Image retrieved from maps.google.com)

\section{Data Collection}

For both data collection sets, the initial setup was the same. The Mavic Pro had a paperclip arrow taped to the side of the fuselage (out of the way of any sensors or moving parts), with the tip pointing towards the ground (Figure 4). The arrow tip allowed a standardized position to be marked/verified before takeoff, and then a data point marked after landing. Each set of points were measured using a traditional tape measurer. The arrow was placed halfway between the two downward vision sensors and offset to the right of the centerline by one inch. The downward vision sensors captured an image of the terrain during a precision takeoff (DJI, 2017). The one-inch offset point tried to mimic the center point assumedly used by the Mavic Pro when calculating a designated point for landing. 


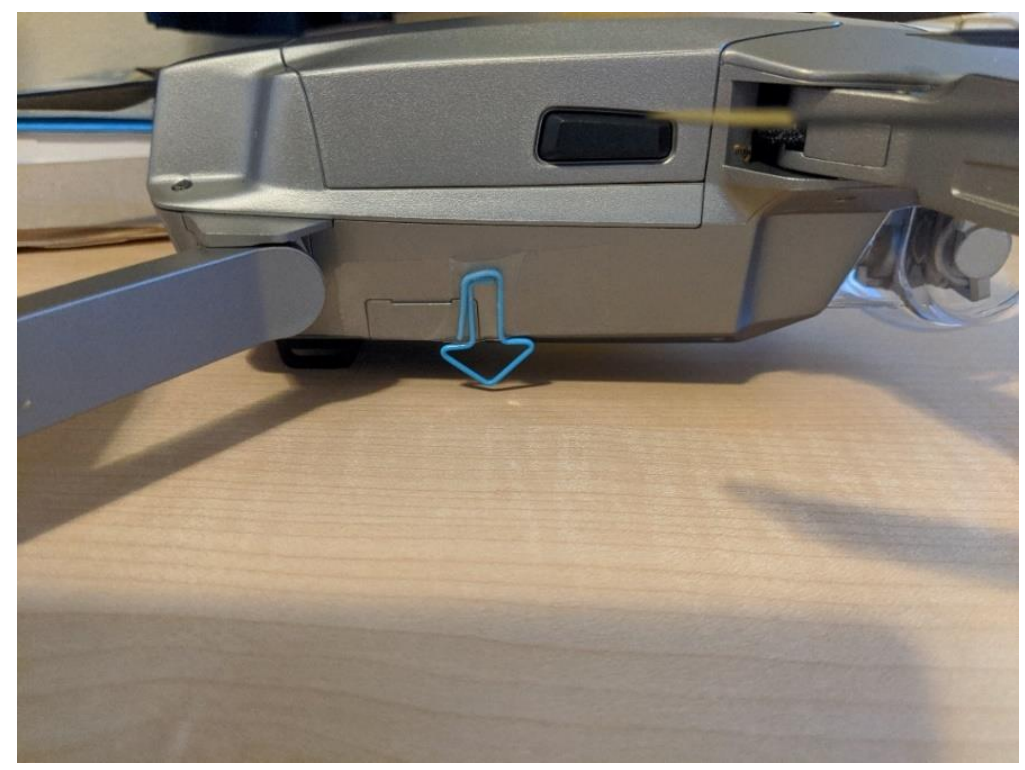

Figure 4. The DJI Mavic Pro with the attached paperclip taped halfway between the downward vision cameras on the right side of the aircraft.

\section{Landing Platform Design}

A simple target recorded the different landing points of the Mavic Pro (Figure 5). The overall launchpad consisted of the 8.5" x 11 " paper target taped to a large (4' x 6') cardboard sheet that allowed for a smooth, firm surface for takeoff/landing operations. This design provided a clean platform to record takeoff/landing data while providing a safe operating platform for the Mavic Pro. The target also created unique terrain by breaking up the nondescript background image of grass, necessary for feature extraction of the precision landings (DJI, User Manual, 2017). Two clean, identical targets were required to record data, one for precision takeoff and landings and the other for non-precision takeoff and landings. 


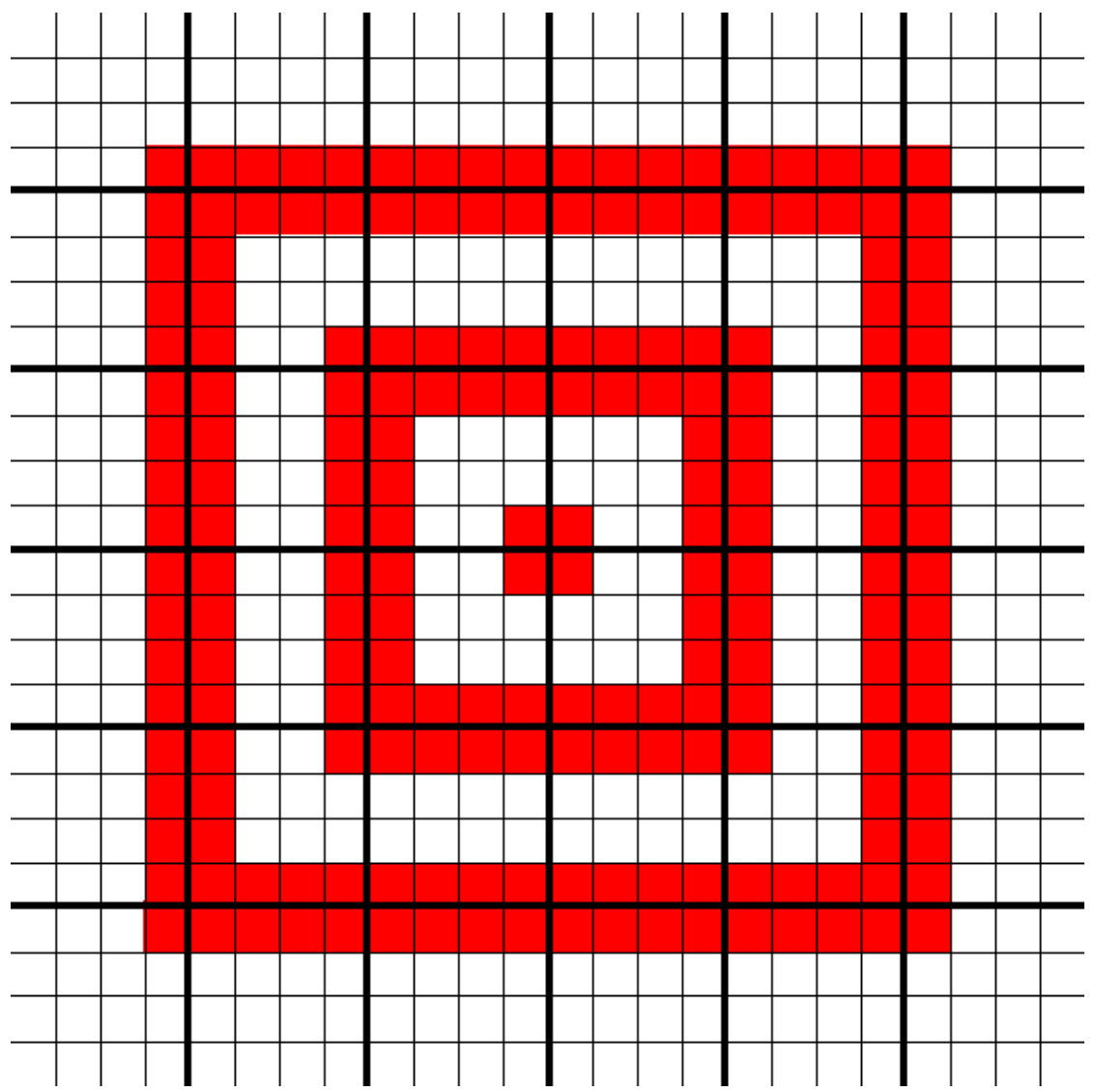

Figure 5. Data was collected using this ground target template.

The standardized takeoff position had three designated points: two front leg points and one point under the paperclip arrow (Figure 6). The arrow marking consisted of dark purple Sharpie located at $(1,0)$. This marking was offset by one inch to the right, implying that the center of the aircraft is over $(0,0)$. Two dark purple circles were traced around the front leg posts, creating a starting position for each front leg. These three points mark the starting takeoff position, accounting for localization and yaw considerations; using the front two legs as reference points prevent any yaw discrepancies. 


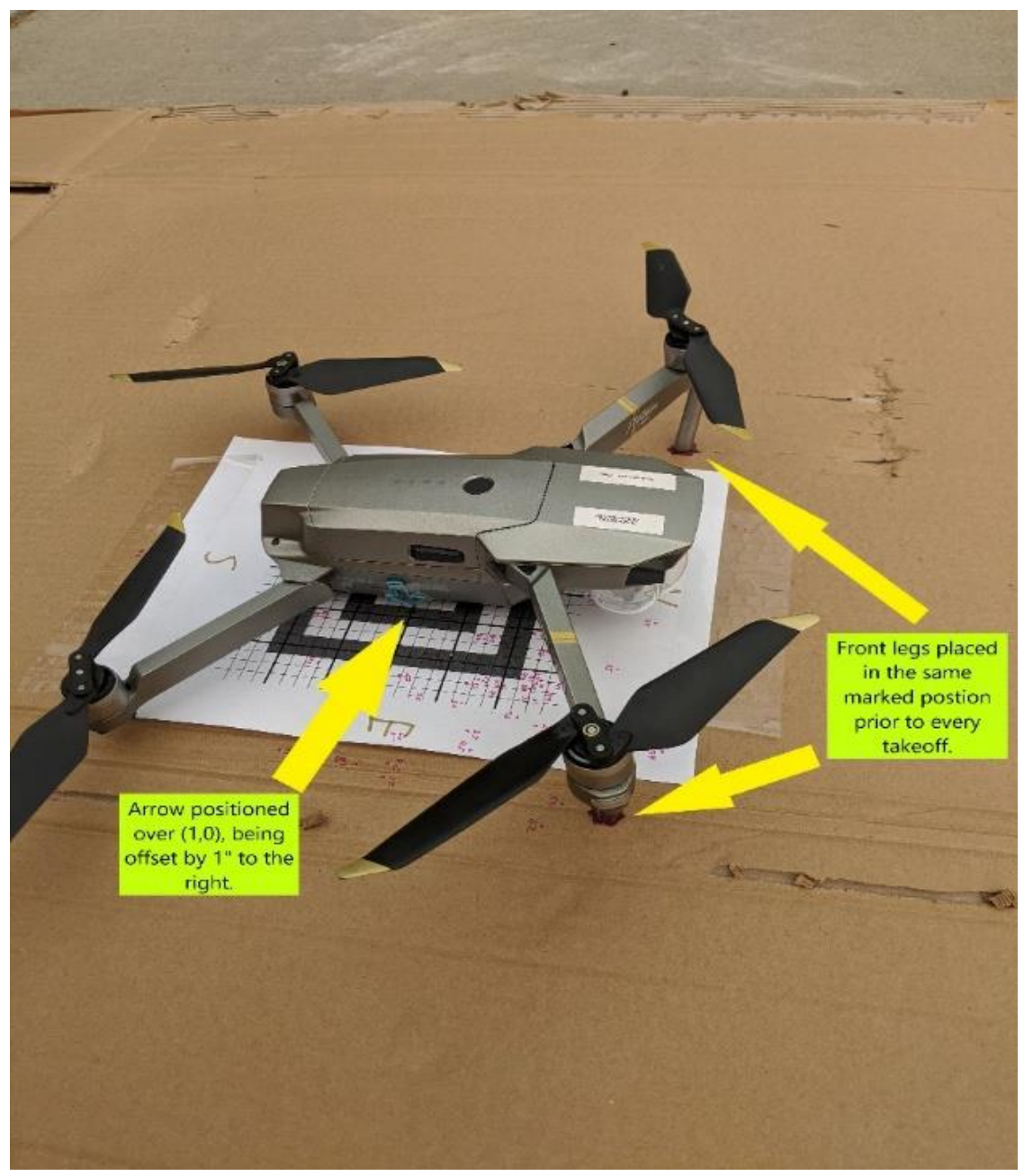

Figure 6. The Mavic Pro is positioned in the same location before every takeoff, with the front legs stationed in marked circles and the paperclip arrow positioned over the same mark at $(1,0)$.

\section{Precision Landing ON}

The DJI GO4 application commanded the "precision landing" takeoff. Sufficient lighting and an area free of obstacles were required (DJI, 2017). Upon activation, the Mavic Pro started its motors and ascended to 19.6 feet (Figure 1). The ascent took 12 seconds to reach 19.6 feet. Once the aircraft reached 19.6 feet, a "smart return-to-home" command was manually activated. The "Smart Returnto-Home" commanded the UAS to return to the Return-to-Home point, where the aircraft then started an autonomous landing (DJI, 2017). Each flight took 12 seconds to ascend to 19.6 feet, with a total round trip taking 40 seconds. 


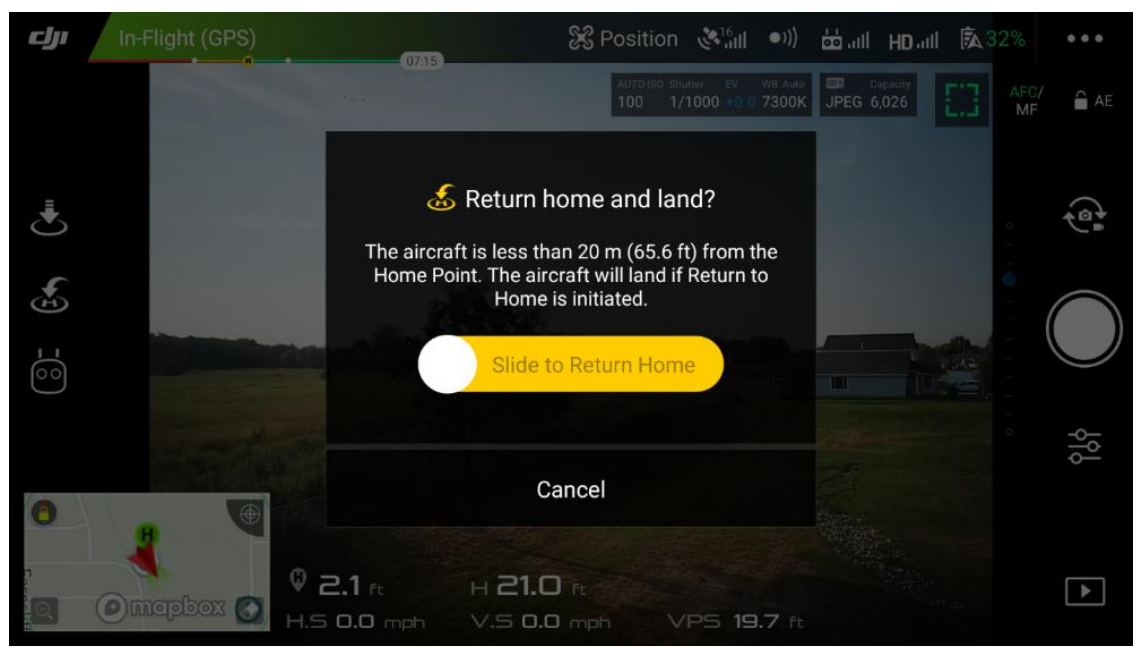

Figure 7. Screenshot of the DJI GO4 Smart Return-to-Home notification (Image retrieved from DJI GO4).

\section{Precision Landing Off}

Autonomous takeoffs and landings kept the two flight profiles (precision landings on/off) similar. The GO4 application "Auto Takeoff" commanded the aircraft to start its motors and ascend to 3' 11" (DJI, User Manual, 2017). The Mavic Pro was then manually flown to 19.6 feet, with all efforts taken to make the ascent take 12 seconds. The "Smart Return-to-Home" command activated the autonomous landing. Each flight profile also lasted approximately 40 seconds round trip to remain consistent with conditions encountered in "precision landing on" flights.

\section{Data Collection}

Data was collected in the same manner for both flight profiles. Once the aircraft landed, a dot and the landing number iteration mark was placed on the target (Figure 8). The aircraft was then reset to the takeoff position for the next iteration. The distance between the arrow's starting position to the designated landing position was determined after completing 64 takeoff and landings. 


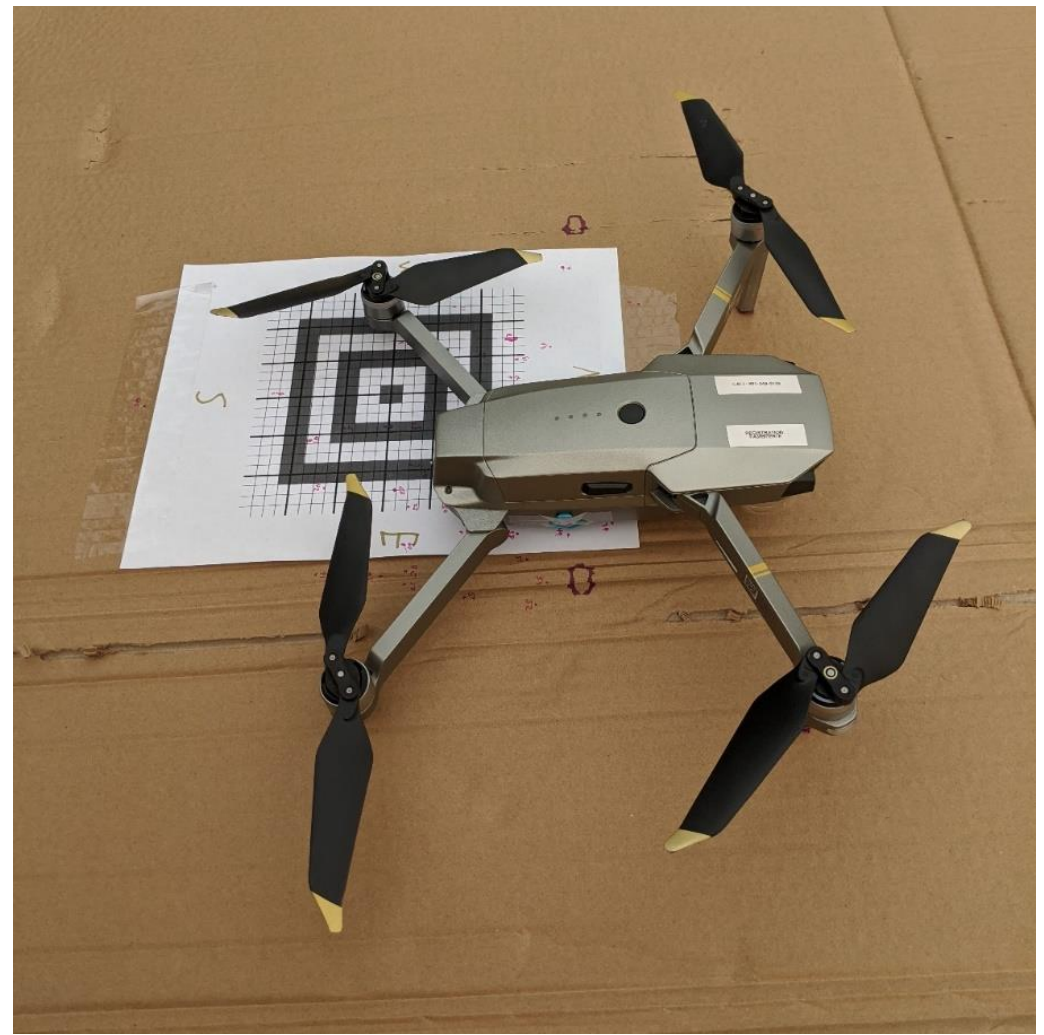

Figure 8. An example of a marked landing point after the drone motors stopped.

\section{Results}

The precision landing-on (PLON) flights flew on August 21, 2020 between 0830 and 0936. The weather reported at the Caldwell, ID airport indicated calm winds, clear skies, a temperature of 20 degrees Celsius, a dew point of 15 degrees Celsius, with an atmospheric pressure of 30.17 " of mercury. The precision landingoff (PLOFF) flights were conducted on August 22, 2020, between 0830 and 0931. The weather reported at the Caldwell, ID airport indicated calm winds, clear skies, a temperature of 19 degrees Celsius, a dew point of 12 degrees Celsius, and an atmospheric pressure of 30.01 " of mercury.

The data presented statistical evidence to reject the null hypothesis indicating there was a statistical performance in mean landing accuracy with PLON $(M=3.45, S D=1.30)$ over PLOFF $(M=4.40, S D=1.89), t(109)=-3.313, p=$ 0.0013 . A mean difference of 0.95 inches represented an improvement in landing accuracy when the precision landing was activated.

A one-tail one-sample t-test was conducted for Hypothesis 2 to determine if the DJI Mavic Pro was capable of wireless charging by landing within $100 \mathrm{~mm}$ (3.937 inches). The PLON landing accuracy $(M=87.63 \mathrm{~mm}, S D=33.02 \mathrm{~mm})$ was 
less than $100 \mathrm{~mm}, t(62)=-2.98, p=0.002$. The $p$-value was significant. There was sufficient evidence to reject the null hypothesis; autonomous landing enabled a Mavic Pro to land within $100 \mathrm{~mm}$ of a designated landing location required for wireless charging.

\section{Conclusion}

Regarding RQ1, the significant $p$-value of 0.0013 suggested there was enough evidence to reject the null hypothesis; PLON increases landing accuracy when compared to PLOFF. The increase in accuracy is approximately one inch (0.95"), with a $95 \%$ CI allowing for landing improvement between 0.384 and 1.529 inches. As depicted in Figure 9, it was interesting to note that PLON tended to provide grouped data points to the top-right, Northeast quadrant of orientation, where PLOFF was more scattered.

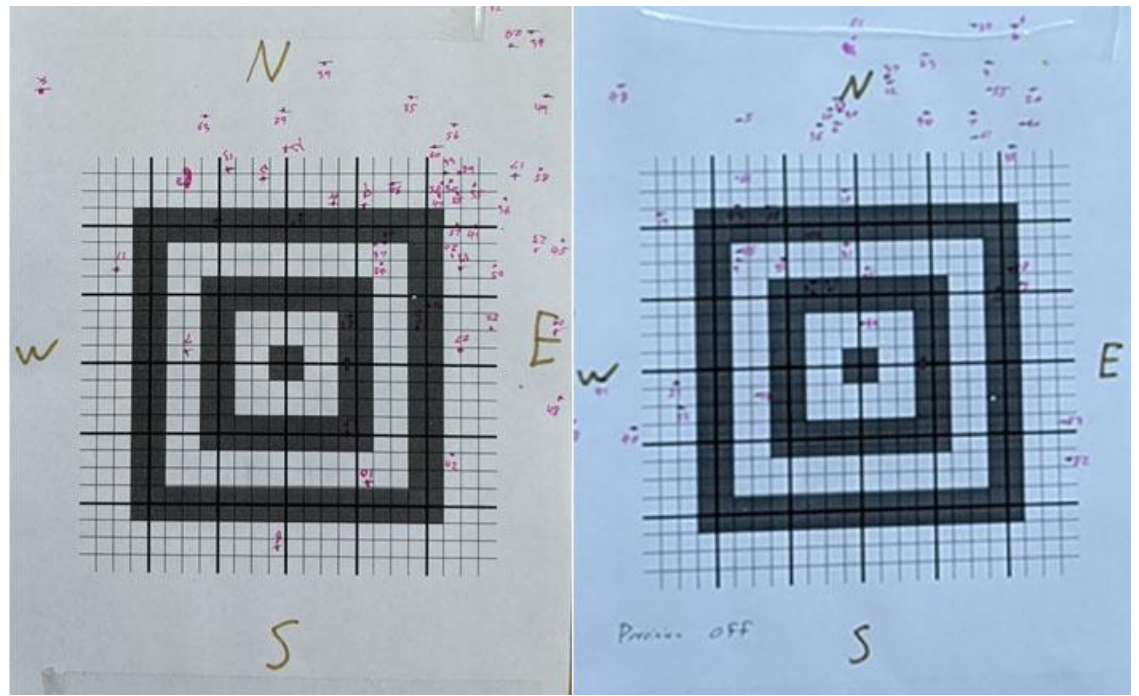

Figure 9. Images depicting the data points for both PLON (left) and PLOFF (right) flights.

Regarding RQ2, with a significant $p$-value of 0.002, there was enough evidence to reject the null hypothesis; the Mavic Pro (PLON) was capable of landing within $100 \mathrm{~mm}$ required for wireless charging. A $95 \% \mathrm{CI}$ allowed for landing within $79.34 \mathrm{~mm}$ to $95.94 \mathrm{~mm}$. There could have been an efficiency level of $88 \%$ in wireless charging with no misalignment, where $83 \%$ efficiency occurred with a $100 \mathrm{~mm}$ misalignment (Rohan et al., 2018). The data provided that there may be some loss in efficiency due to approximately $5 \%$ of misalignment. Wireless charging systems, landing within designated spaces in emergencies, and landing on small platforms are within the realm of possibilities with the DJI Mavic Pro used for this research. The exact algorithm that DJI uses for precision landings is still 
publicly unknown; however, these results demonstrate the potential for future applications.

\section{Recommendations}

Future research should try to replicate the results of this project. Doing so would determine if other UAS, different locations, and atmospheric conditions would have different results. There should be changes made in the described method with future research. Incorporating a flight pattern with varying distances and vectors after takeoff, then commanding the autonomous return-to-home and landing could provide different results. Research should be conducted into the accuracy when landing with varying background by removing the landing $\mathrm{pad} /$ target. Other times of day, with different lighting and weather conditions, could also provide valuable insight. The results of the recommended research would strengthen or neutralize the efficacy of precision landings. 


\section{References}

Borowczyk, A., Nguyen, D., Nguyen, A. P., Nguyen, D. Q., Saussié, D., \& Ny, J. L. (2017). Autonomous landing of a quadcopter on a high-speed ground vehicle. Journal of Guidance, Control, and Dynamics, 40(9), 2378-2385. doi:10.2514/1.g002703

DJI. (2017, December). Mavic pro: User manual. Retrieved from https://dl.djicdn.com/downloads/mavic/Mavic_Pro_User_Manual_v2.0_en .pdf

51 Drones (Director). (2018, 26 October). DJI precision landing | IMU calibration why and how? [Video file]. Retrieved from https://www.youtube.com/watch?v=OXD0qWV96LM

Kaljahi, M. A., Shivakumara, P., Idris, M. Y., Anisi, M. H., Lu, T., Blumenstein, M., \& Noor, N. M. (2019). An automatic zone detection system for safe landing of UAVs. Expert Systems with Applications, 122, 319-333. doi:10.1016/j.eswa.2019.01.024

Kyristsis, S., Antonopoulos, A., Chanialakis, T., Stefanakis, E., Linardos, C., Tripolitsiotis, A., \& Partsinevelos, P. (2016). Towards autonomous modular UAV missions: The detection, geolocation and landing paradigm. Sensors, 16(11), 1844. doi:10.3390/s16111844

Miller, B., Miller, A., Popov, A., \& Stepanyan, K. (2019). UAV landing based on the optical flow videonavigation. Sensors, 19(6), 1351. doi:10.3390/s19061351

Nguyen, P. H., Kim, K. W., Lee, Y. W., \& Park, K. R. (2017). Remote markerased tracking for UAV landing using visible-light camera sensor. Sensors, 17(9), 1987. doi:10.3390/s17091987

Nguyen, P., Arsalan, M., Koo, J., Naqvi, R., Truong, N., \& Park, K. (2018). LightDenseYOLO: A fast and accurate marker tracker for autonomous UAV landing by visible light camera sensor on drone. Sensors, 18(6), 1703. doi:10.3390/s18061703

Rodriguez-Ramos, A., Sampedro, C., Bavle, H., Puente, P. D., \& Campoy, P. (2018). A deep reinforcement learning strategy for UAV autonomous landing on a moving platform. Journal of Intelligent \& Robotic Systems, 93(1-2), 351-366. doi:10.1007/s10846-018-0891-8

Rohan, A., Rabah, M., Talha, M., \& Kim, S. (2018). Development of intelligent drone battery charging system based on wireless power transmission using hill climbing algorithm. Applied System Innovation, 1(4), 44. doi:10.3390/asi1040044

Wang, L., \& Bai, X. (2017). Quadrotor autonomous approaching and landing on a vessel deck. Journal of Intelligent \& Robotic Systems, 92(1), 125-143. doi:10.1007/s10846-017-0757-5 
Yang, Q., \& Sun, L. (2018). A fuzzy complementary Kalman filter based on visual and IMU data for UAV landing. Optik, 173, 279-291. DOI:10.1016/j.ijleo.2018.08.011

Zhang, C., \& Kovacs, J. M. (2012). The application of small unmanned aerial systems for precision agriculture: A review. Precision Agriculture, 13(6), 693-712. DOI:10.1007/s11119-012-9274-5

Zhong, X., Peng, X., Yan, S., Shen, M., \& Zhai, Y. (2018). Assessment of the feasibility of detecting concrete cracks in images acquired by unmanned aerial vehicles. Automation in Construction, 89, 49-57. DOI:10.1016/j.autcon.2018.01.005 